\title{
Configurações
}

Revista de sociologia

\section{Nanotecnologia e videojogos: contestar a narrativa do Norte a partir das Epistemologias do Sul}

Nanotechnology and Video games: Challenging the Narrative of the North with Epistemologies of the South

Nanotechnologie et jeux vidéo: défier la narrative du Nord avec les

épistémologies du Sud

\section{Rui Vieira Cruz}

\section{(2) OpenEdition}

\section{Journals}

Edição electrónica

URL: http://journals.openedition.org/configuracoes/2168

DOI: $10.4000 /$ configuracoes. 2168

ISSN: 2182-7419

\section{Editora}

Centro de Investigação em Ciências Sociais

\section{Edição impressa}

Data de publição: 1 Dezembro 2013

Paginação: 199-218

ISBN: 1646-5075

ISSN: 1646-5075

\section{Refêrencia eletrónica}

Rui Vieira Cruz, « Nanotecnologia e videojogos: contestar a narrativa do Norte a partir das

Epistemologias do Sul », Configurações [Online], 12 | 2013, posto online no dia 19 novembro 2014, consultado o 30 abril 2019. URL : http://journals.openedition.org/configuracoes/2168 ; DOI : 10.4000/ configuracoes. 2168

Este documento foi criado de forma automática no dia 30 Abril 2019. 


\title{
Nanotecnologia e videojogos: contestar a narrativa do Norte a partir das Epistemologias do Sul
}

\author{
Nanotechnology and Video games: Challenging the Narrative of the North with \\ Epistemologies of the South \\ Nanotechnologie et jeux vidéo: défier la narrative du Nord avec les \\ épistémologies du Sud
}

Rui Vieira Cruz

\section{Introdução: Epistemologias do Sul, nanotecnologia e videojogos}

1 A identificação da ciência uniformizadora e positivista produzida pelo Norte global como um produto do Iluminismo, aliada às formas de organização científica do trabalho, contribuiu largamente para a eliminação de outras formas de encarar a ciência, as suas epistemologias, os seus paradigmas e as suas metodologias. Um dos efeitos foi o reforço do pensamento do Norte, construído internamente em categorias dicotómicas mutuamente exclusivas e abissais a partir do berço da ciência helénica de Parménides: Realidade-Aparência, Espesso-Fino, Quente-Frio, Luz-Sombra, Ser-Não ser, Norte-Sul, Masculino-Feminino, Humano-Subhumano, Céu-Inferno, Mito-História, Certo-Errado. Como consequência dessa construção dicotómica do mundo na qual tudo tem de ser inserido em categorias opostas - eventos coletivamente exaustivos -, é amplamente ignorado que ambos os polos regularmente se intersectam. Desta forma, as culturas dominantes que fomentaram o aparecimento metafórico do Norte criaram uma forma monocultural do saber e do rigor do saber - um sistema hegemónico que exclui, marginaliza e subalterniza as formas de conhecimento que não assentam na sua idiossincrasia -,produzindo, por defeito, metaforicamente o Sul (Santos, 2008). Este Sul, sistema periférico na produção e validação de conhecimento, é tido como alternativo e 
posto de parte, fruto da colonização de poder (Grosfoguel, 2008). Assim, torna-se necessário resgatar o debate epistemológico (Nunes, 2008), aliando-o à diversidade de saberes. Cabe às Epistemologias do Sul a demonstração da impossibilidade de uma epistemologia geral padronizada e ditada através de um processo hegemónico (Santos, 2008). Ora, nas diferentes indústrias culturais tal também se reflete: são diversos os trabalhos realizados na literatura e no cinema sobre o poder hegemónico do Norte e sobre a incorporação do Sul na construção identitária das narrativas (Arruda, 2012). Porém, o universo dos videojogos e da nanotecnologia é uma área onde não abundam os estudos. Assumir que a identidade dos objetos técnicos, como os nanotecnológicos, é apenas construída pelo domínio da ciência e tecnologia não tem em conta a capacidade de veiculação das indústrias culturais. A forma como os indivíduos ouvem, vêm e interagem com os objetos técnicos contribui para lhes conferir uma identidade.

2 Assumindo que a "identidade é acima de tudo autodefi nição [...] elaborada em oposição ou contraste com heterodefinições, processo no qual se demarcam fronteiras de pertença e exclusão" (Ribeiro, 2011: 33), tal remete para uma ligação dual para a criação do "eu" e do "outro". Fruto da ação hegemónica do "Norte", ocorre o efeito de o "Sul" não se olhar pelos seus próprios olhos, à luz dos seus princípios, conhecimentos e saberes, mas ver-se pelos olhos do outro. Este princípio de não existência mina a legitimidade das suas formas de conhecimento, das suas mundividências, focando-as nos parâmetros de análise do agente hegemónico. As identidades, longe de estáticas e reificadas, não são inamovíveis e previsíveis (Giddens, 2006), mas convertem-se em tal quando coisificadas e protegidas de forma a manter a sua "originalidade".

3 Transpondo para os videojogos, a identificação dos jogadores pelos seus avatares e a sua incorporação/identificação com as personagens fomentam princípios de alter-identidade (Li, Liau \& Khoo, 2013). Assim, se no universo dos videojogos, meio em franca expansão (Marchand \& Hennig-Thurau, 2013) mas ainda na periferia cultural, forem apenas emitidas personagens, cenários e condições produzidos pelo Norte global, mas consumidos quer pelo Norte quer pelo Sul, veicula-se uma forma de dominação cultural na qual o Sul é manifestamente o recetáculo da produção cultural criada no Norte.

Como não tremer de impaciência perante a ideia do mundo que nos espera: Os mesmos filmes, as mesmas séries televisivas, as mesmas informações, as mesmas canções, os mesmos slogans publicitários, os mesmos objectos, a mesma roupa, os mesmos automóveis, o mesmo urbanismo, a mesma arquitectura, o mesmo tipo de apartamentos, muitas vezes mobilados e decorados da mesma maneira [...] fulminante ofensiva da estandardização, da homogeneização, da uniformização [...] é McDonalds e Planeta Disney a nível mundial. A apropriação, a mutilação e a sensaboria imbecil do património dos outros, Hercule, Notre Dame de Paris... será isto uma interfecundação cultural destinada a contribuir para o enriquecimento espiritual da humanidade? (Passet, 2002: 105)

Esta forma de dominação, não mais assente nos princípios de colonização que marcaram a Era Moderna, fomenta o seu modelo de racionalidade (ocidental) que se assume como superior a todas as outras formas de conhecimento. Assim, a legitimação da constante subalternização, quer moral quer social, das restantes formas de conhecimento conduz a necessidade da constante supervisão dos contributos das restantes formas de conhecimento não tidas como racionais. O domínio da produção do Norte reforça a sua posição hegemónica impondo-se científica e moralmente sobre as restantes instâncias culturais, organizando o mundo de acordo com a sua cosmovisão, relegando para segundo 
plano, subalternizando, marginalizando e atribuindo um não ser, uma não existência ao "outro", no qual se insere a metáfora do Sul (Khan, 2011).

5 A investigação das ciências sociais, em particular a ligada aos estudos culturais, não tem focado regularmente o mundo dos videojogos. São vários os autores (Bornet, 2012; Heidbrink et al., 2014) que focam o fenómeno religioso centrado no cristianismo europeu como o principal responsável pelo afastamento do estudo dos jogos. Tal deve-se à abolição do romano ludi da doutrina teológica cristã, pela sua consideração imoral e ligações às culturas pagãs, tendo a reforma protestante e as práticas católicas romanas reforçado a condenação dos jogos (de azar e apostas). Somente a partir dos anos 70 do século $\mathrm{XX}^{1}$ é que os jogos se tornaram parte integrante dos estudos académicos e do meio empresarial. Tendo o seu foco comercial em países como o Japão e os Estados Unidos, assim como os países europeus, a abordagem das diversas produtoras e editoras às referências geoestratégicas tem sido uma das esferas mais relegadas de análise académica. Não obstante o seu valor económico crescente, a forma como o tema é analisado pertence ainda à periferia da cultura mainstream ${ }^{2}$ (Carbone e Rufino, 2013).

Por sua vez, a questão da identidade retratada através da (nano)tecnologia não parece ser alvo de debate teórico nas ciências sociais, dirigindo o seu foco para a transformação que a nanotecnologia possibilita em termos socioeconómicos (Wood, Jones \& Geldart, 2007), filosóficos (Dupuy, 2010), religiosos (Toumey, 2011) e ético-morais (Ferrari, 2010) na relação humano-máquina. Todavia, a conversão da tecnologia num objeto autónomo de análise inserido num quadro de referência não se manifesta como a norma. Se "o nosso ser deixa de ser Dasein[3], quando adotamos o ser de identidades prontas-a-existir" (Gibbs in Araújo, 2011: 9), os videojogos, como em muitas das indústrias culturais, assentam na produção localizada no espaço e no tempo de objetos comercializados à escala global.

7 Os trabalhos desenvolvidos para tentar compreender como a nanotecnologia é retratada não abordam prioritariamente os conteúdos, centrando-se essencialmente sobre as perceções e formas de compreensão de diversos atores em torno do tema (Macnaghten, Kearnes \& Wynne, 2005; Macoubrie, 2006; Nordmann \& Macnaghten, 2010; Marchant, 2008; Katz, 2009; Kurath, 2009; Kuzma, 2007).

8 Pretendemos, por isso, analisar a narrativa e os elementos de jogabilidade em videojogos que atribuem um papel central à nanotecnologia, tendo como eixo analítico a sociologia da ciência e tecnologia, mas analisando a problemática a partir das Epistemologias do Sul.

\section{Modernidade, Iluminismo e a Era da Razão}

9 A história humana tem sido pródiga na definição de elementos de identidade e heteroidentidade. Desde a Dinastia Han na China e do Império Romano que a noção identitária de estar dentro das fronteiras geográficas do império se constrói em oposição a estar do lado de fora das fronteiras, cabendo a qualquer um destes sistemas a função de expansão através da conquista militar do território do outro. Muitos elementos simbólicos foram assim criados, tais como bandeiras, brasões, moedas e um exército (Bourdieu, 2001) sob o jugo de um comando central. No quadro referente ao início da Idade Moderna, período iniciado pela tomada de Constantinopla (1453) e pela expansão gerada após os Descobrimentos portugueses e espanhóis, estabeleceram-se dois eixos analíticos nos quais se envolvem as relações entre o descobridor e o descoberto. Se de um lado nos deparamos 
com o agente "móvel" e portanto expansionista e global, do outro é-nos retratado o agente "estático", aquele que é local, autóctone, um mero recetáculo (Appadurai, 1988). Assim, enquanto o agente móvel sai geograficamente do seu espaço de conforto, deslocando-se em direção ao desconhecido de forma a conhecer e dominar novos espaços, o agente estático representa o "outro", aquele confinado às suas raízes, tradições e culturas. É esta construção epistemológica que permite ao primeiro manter uma posição central no sistema-mundo, cabendo ao segundo resignar-se com a sua posição periférica (Wallerstein, 1999).

10 Com a propagação do Iluminismo (Era da Razão) levada a cabo por diferentes países da Europa Central, em particular a França, Inglaterra, Países Baixos e Alemanha, a importância dos países do Sul da Europa, em particular Grécia, Itália, Portugal e Espanha, outrora elementos centrais na construção da identidade europeia, foram sendo redesenhados, reconvertendo os papéis deste conjunto de países. Esta cosmovisão promove o Sul como o centro da história passada (a arte grega, o Império Romano, os descobrimentos portugueses e a dominação espanhola), mas ocupando um papel residual e mais periférico nos parâmetros geopolíticos e culturais atuais.

11 A tese da economia clássica focada nos ritmos e nos estádios de desenvolvimento (Solow, 1979) como um processo de evolução linear cria a configuração de que os países desenvolvidos devem ajudar os outros a desenvolver-se, concebendo a perspetiva de que, ao serem interiorizados os valores culturais da civilização, todos poderão atingir um mesmo estádio de desenvolvimento. Tal conceptualização ideológica revela a superiorização de uma forma cultural sobre as restantes, retirando ao "outro" a sua própria racionalidade e a sua autoidentidade, mas sobretudo retirando legitimidade às suas aspirações. Essas formas de violência (de cariz simbólico e/ou material) atravessaram a História, exprimindo-se através de formas de dominação como a escravatura, o genocídio e a exploração (novamente simbólica e material), propagando o "outro" como ontológica e epistemologicamente inferior.

\title{
3. Nanotecnologia: o objeto de análise
}

12 São variadas as definições em torno do conceito de nanotecnologia oscilando entre os ramos da ciência e tecnologia que trabalham com objetos mais pequenos do que um mícron (1000 nanómetros) ou como uma medida de entre 1 e 100 nanómetros (nm).

\begin{abstract}
How small does a man-made structure need to be before we can count it a nanotechnology? A (successful) candidate for a job at my university was asked this question; he replied with fi rm authority, '100 nanometers'. Why 100? the panel asked, to which he replied without a moment's hesitation, 'Because President Clinton[ $\left.{ }^{4}\right]$ says so.' This seemed a very fair answer $-100 \mathrm{~nm}$ was in indeed the fi gure associated with the USA's National Nanotechnology Initiative, and the invocation of the authority of the US president pretty much sums up the arbitrary nature definitions like this. (Jones, 2007: 38-39)
\end{abstract}

13 Esta tensão/problematização na construção (social) do objeto técnico (Neves, 2006; Simões, 2005) em vários sectores (político, social, industrial...) tem levantado uma série de questões sobre como a tecnologia é formada e os efeitos derivados da sua aplicação.

Se para Roco (2007) a nanotecnologia é o pivô central no desenvolvimento económico e social, para Bainsbridge (2007),no futuro próximo ela é de vital importância para as economias do conhecimento do século XXI. Assim, é possível encontrar a nanotecnologia 
em diversos sectores de aplicação, em particular os sectores energético, de produção alimentar, da saúde, militar entre outros (Singer, 2005; Smalley, 2007). Também a sua ligação a outras tecnologias, nomeadamente as ciências da computação e a biotecnologia, veio ampliar o alcance de aplicações assentes na nanotecnologia, em especial na formulação e transformação de materiais. Podemos discutir assim a formação de novos paradigmas tecnológicos convergentes com base fomentada pela nanotecnologia, em particular GNR (Genética, Nanotecnologia, Robótica), BANG (Bio, Átomos, Nano e Genética) e NBIC (Nano, Bio, ciências da Informação e ciências Cognitivas).

15 A importância da nanotecnologia enquanto projeto de desenvolvimento (BensaudVincent, 2010), com ligação aos sectores de aplicação e um vasto leque de tecnologias, faz dela um componente central neste artigo, em concreto, a sua representação num conjunto de videojogos.

\section{Da jogabilidade ao storytelling: a especificidade narrativa dos videojogos}

Os atenienses separavam os seus espaços, criando, de um lado, o local onde praticavam a política - o pnyx - e do outro o centro económico da cidade - a ágora. Se a intenção parecia evidente - separar a atividade política da atividade económica (Sennet, 2007) -, também os videojogos possuem a capacidade de demarcarem a sua vertente artística da sua vertente comercial. Desde as suas possibilidades gráficas aos sistemas de jogabilidade e às narrativas que possuem, são vários os elementos que provocam reações no jogador. Os eixos de jogabilidade e de narratologia rapidamente se tornaram os focos centrais das análises. Mas há outros aspetos que devemos ter em conta.

Durante um longo período de tempo da humanidade, o ato de contar histórias era uma atividade presencial. Enquanto alguém narrava uma história, do outro lado estava alguém que ouvia, respondia e interagia em função dos conteúdos e da forma como era feita a narrativa. Esta poderia, assim, ser recontada por outros indivíduos, sofrer transformações, ver acrescentadas ou eliminadas partes, sem se conseguir defi nir onde e quando essa transformação ocorreu. De acordo com Gerbner (1995), era este processo que tornava o storytelling ajustável, responsivo e participativo, servindo também como ritual de passagem, no qual o indivíduo ia transitando de ouvinte passivo a reprodutor e criador de conteúdos.

Com a escrita veio uma maior consistência na criação da narrativa. Contudo os elevados índices de analfabetismo, o árduo trabalho de copiar os exemplares originais e os problemas de tradução linguística faziam com que uma esmagadora maioria de indivíduos não acedessem aos conteúdos originais. A criação da prensa de Gutenberg configurou um novo leque de possibilidades: permitia a diversas comunidades espalharem uma história de uma forma mais direta. Com a evolução da imprensa, após a revolução industrial nasceu o público de massas. A imprensa tornava-se assim a forma de transmitir uma mesma mensagem a milhares ou milhões de indivíduos que, à partida, não teriam nada em comum.

19 Esta é uma questão central: como propagar uma mensagem a indivíduos que não possuem nada em comum, que partilham os interesses de diferentes classes ou segmentos sociais e que possuem mundividências radicalmente distintas? A "liberdade de imprensa" foi a forma como se operacionalizou tal ação: diferentes meios possuem diferentes formas de 
contar as histórias. Com o avanço da "sociedade de massas", esta começou a monopolizar a cultura para as suas finalidades, pelo que a integração e formas de compreensão da cultura passaram a contribuir para uma determinada posição de classe e status (Arendt, 1968). Os fenómenos culturais tornam-se distintivos e, consoante as práticas culturais efetuadas, é possível perceber e catalogar a posição social de um determinado indivíduo ou conjunto de indivíduos (Bourdieu, 1999; 2011).

À medida que a imprensa evoluía, com ela surgiam outras formas paralelas de contar histórias. A televisão foi uma das inovações que, ao mesmo tempo que retribalizaram o storytelling, também foram capazes de criar conteúdos universais para diferentes pontos do planeta. São assim focados dois pontos essenciais na evolução da forma como uma narrativa é contada. Primeiro, a evolução dos meios de comunicação não é linear: a imprensa não se esgota aquando do aparecimento da TV, nem a TV se esgota aquando do aparecimento da internet. Os sistemas fundem-se e transformam-se. A sua evolução depende assim mais da forma como os produtores e consumidores de conteúdo interagem do que da evolução tecnológica em si. Segundo, o consumo televisivo é essencialmente ritualístico. Mais do que dos conteúdos transmitidos, a televisão depende dos horários de consumo. Em suma, ela depende da forma como os indivíduos organizam o seu quotidiano e dos espaços temporais que têm disponíveis.

Os videojogos representam então um regime de representação em que é possível sair desta lógica, uma vez que o jogador tem a possibilidade de seguir um percurso não linear. Embora sempre determinado pelos elementos criados pelas produtoras e editoras de videojogos, a existência de múltiplos percursos que se ligam a múltiplos finais abre a possibilidade de escapar a uma lógica de história única e a um percurso e visão impostos e lineares da narrativa. Atendendo à telepresença (Minsky, 1980), um estado de consciência que confere a impressão de estar fisicamente presente num mundo mediado, bem como o sentimento subjetivo de imersão num ambiente virtual (Weibel et al., 2008), atualmente, com o advento da internet e a reprodução de verdadeiras economias dentro de um jogo ${ }^{5}$, os jogadores estabelecem um conjunto de relações sociais e económicas assentes em pressupostos reais no mundo virtual - e.g., de Azeroth, o mundo de Warcraft (Billieaux et al., 2013) -, mas procedem à criação de novos códigos linguísticos (Decker \& Gay, 2011) e novas normas e relações (Yang, 2012). Tal deve-se à incorporação do jogador na personagem, seja ela o seu avatar (imagem virtual) ou não (Li et al., 2013).

\section{Videojogos: o efeito da jogabilidade}

São vários e diversificados os meios pelos quais os indivíduos acedem a um conjunto de elementos culturais. Os que por norma constroem uma história/narrativa pertencem ao universo da literatura, filmes, séries televisivas e videojogos. Enquanto a literatura permite aos leitores construírem mentalmente cenários, constituindo-se cada livro como um universo pessoal, a TV é capaz de transmitir um conjunto de mensagens assentes na realidade (por exemplo, telejornais) e, ao mesmo tempo, envolver os seus telespectadores num conjunto de universos imaginados através de séries e filmes. Por seu lado, os videojogos revelam um nível de interatividade não presente nem na literatura, nem nos filmes ou nas séries, uma vez que nestes o indivíduo não controla os destinos da sua personagem ou da narrativa (Zagalo, 2009).

In literature, theatre and fi $\mathrm{lm}$ everything matters or is conventionally supposed to matter equally - if you've seen $90 \%$ of the presentation that's not enough, you have 
to see or read it all (or everything you can). This is characteristic of dominantly interpretative practices in general. In contrast, in computer games you either can't or don't have to encounter every possible combinatory event and existent the game contains, as these differ in their ergodic importance. (Eskelinen, 2001: 5) editoras, suscita uma outra questão: ao contrário do cinema, por exemplo, os videojogos não dependem do fotorrealismo, ou seja, não estão limitados na sua capacidade criativa. Novos mundos, novas físicas (e.g., a gravidade não é condição basilar), novas realidades (e.g., universos paralelos) são elementos com uma forte vertente de contestação e que perturbam a estrutura e a realidade vigentes. Da mesma forma, as condições sociais impostas servem igualmente como forma de contestação da hegemonia. Se títulos como Bioshock (shooter) desafiam filosoficamente o objetivismo (assente em Ayn Rand), jogos como Gone Home (novela gráfica) colocam efetivamente o jogador na pele de uma mulher queer, enquanto Mass Effect e Dragon Age (ambos RPG) abrem possibilidades a relações homossexuais e até interespécies. Tal como são escassos os videojogos que abordam estes campos, também os trabalhos académicos que relacionam os videojogos com a nanotecnologia são praticamente inexistentes (Milburn, 2011).

\section{Nanotecnologia e videojogos}

A nanotecnologia tem sido regularmente abordada pela indústria dos videojogos ${ }^{8}$. A saga de jogos Metal Gear (com particular destaque para Metal Gear Solid IV e Metal Gear Rising Revengeance) promoveu juntos dos jogadores algumas das grandes potencialidades da nanotecnologia, tal como armas capazes de atacar o inimigo tendo em conta o seu código genético e a capacidade curativa das personagens através de sangue artificial. Aqui existe também uma nanomáquina com inteligência artificial capaz de controlar, regular e melhorar as capacidades de todos os soldados de uma determinada fação. Títulos como Crysis, Crysis 2 e Crysis 3 incorporam nanofatos em atividades militares, enquanto a saga Deus Ex (The Conspiracy e Human Revolution) e a trilogia Mass Effect (Mass Effect, Mass Effect 2 e Mass Effect 3) referem algumas das potencialidades destruidoras das nanotecnologias, mas também algumas das vantagens destas através de nanomelhoramentos, ou nanoenhancements (de que também é exemplo Sudeki). É até possível escolher que áreas se pretendem melhorar com a nanotecnologia, desde o armamento até à inculcação de nanodispositivos cibernéticos no corpo humano que conferem força sobre-humana.

A saga Red Faction (Guerilla e posteriormente Armaggedon) mantém uma estreita ligação entre a nanotecnologia e as suas aplicações militares, nomeadamente pela criação de uma nanoarma (nanorifl e) capaz de destruir estruturas humanas e desintegrar o corpo

Configurações, 12 | 2013 
humano. Neste mesmo jogo a nanotecnologia serve para o controlo da mente humana através de nanodispositivos. Outras produções como Electro Plankton, Kurikin Nano Island Story, Nanosaur Extreme, Nanotek Warrior, Nanostray, Perimeter, Nanobreaker, Eve Online demonstram uma pluralidade de sectores de aplicação e formas de utilização da nanotecnologia de alguma forma ligando a ação militar (Milburn, 2005) com outros campos.

A seleção das plataformas foi efetuada tendo em conta as mais vendidas e aquelas com maior base de jogadores. A geração de consolas atual teve início em $2005 \mathrm{com}$ o lançamento da Xbox360 (Microsoft), sucedendo-se em 2006 a Wii (Nintendo) e em 2007 a PlayStation 3 (Sony). Esta geração teve o seu término em novembro de $2013^{9}$, aquando do lançamento da PlayStation 4 e da Xbox One e após o lançamento da Wii U.

A comparação efetuada incide sobre três franchises ${ }^{10}$ (abarcando seis títulos no total) nos quais a nanotecnologia assume central importância e tem como base o estudo conduzido por Christou (2013) no qual o autor compara um eixo temático em dois estilos diferentes RPG e FPS - e as perceções de jogadores com ou sem experiência.

Atendendo aos géneros preferidos pelos jogadores de acordo com a Entertainment Software Agency (ESA, 2013) - Ação/Aventura, Shooter e Role Playing Game (RPG) -,selecionámos três séries11 que abordassem a questão da nanotecnologia e simultaneamente tivessem atingido um volume de vendas considerável no seu segmento. As escolhas recaíram sobre Metal Gear Solid IV: Guns of Patriots, Metal Gear Rising Reveangeance, Deus Ex: Human Revolution , Crysis, Crysis 2 e Crysis 3. Metal Gear, uma franquia criada em 1987 pela japonesa Konami Computer Entertainment, totalizava, até outubro de 2013, 32,63 milhões de unidades vendidas (VGChartz). As duas entregas12 que compuseram a nossa análise - Metal Gear Solid IV: Gunsof Patriots (5,84M), das nipónicas Kojima Productions (produtora) e Konami (editora), e Metal Gear Rising: Revengeance (1,41M), da Platinum Games (produtora) e da Konami (editora) - perfazem um total de 7,25 milhões de unidades. Em termos globais da saga, a combinação de ambos os títulos perfazem $22,2 \%$ do número de unidades vendidas.

Deus Ex é uma franquia criada em 2000 pela produtora canadiana Eidos Montreal, ligada à empresa franco-canadiana Eidos Interactive (que posteriormente deu origem à Square Enix Europe), e distribuída pela japonesa Square Enix. Até outubro de 2013 tinha contabilizado 4,5 milhões de unidades vendidas, sendo Deus Ex: Human Revolution responsável por 3,02 milhões dessas unidades (VGChartz). Em termos globais da saga, composta por quatro títulos, estes resultados representam $71,1 \%$ do volume de vendas.

31 Crysis é o mais recente dos três franchises analisados. A produtora responsável é a alemã Crytek (estúdios de Frankfurt) e é editado pela americana Electronic Arts. A sua origem data de 2007, tendo sido comercializadas até outubro de 2013 um total de 7,99 milhões de unidades. Os três títulos estudados, Crysis, Crysis 2 e Crysis 3, perfi zeram 3 milhões, 3,21 milhões e 1,78 milhões de cópias vendidas, respetivamente. Em termos globais, a série, composta por três entradas, perfaz $62,5 \%$ do total de unidades vendidas.

No universo dos videojogos, "diversity is not the result of demand by audiences, but is rather the social responsibility of media producers" (Shaw, 2010: 3). Por este motivo centramo-nos nos produtores de conteúdos, em particular nas produtoras e nas editoras de videojogos. Alguns dos dados aqui explanados focam a sua localização geográfica, o tipo de conteúdo desenvolvido especificado pelo género de jogo e, finalmente, a representação. 

produtoras e editoras dos videojogos selecionados. Os videojogos, ao assentarem num conjunto de emoções (Zagalo, 2009), tais como alegria, satisfação, tristeza, frustração derivadas da sua narrativa, mas também na sua jogabilidade, criam a possibilidade de o jogador poder responder positivamente a uma cena moralmente negativa e vice-versa. Novamente, tal resposta pode depender da narrativa, dado que o jogador pode não compreender a representação social por detrás da criação da cena; contudo, pode também focar-se na jogabilidade e não na narrativa. Esta vertente culturalmente específica e culturalmente contingente dos videojogos abre, no entanto, uma nova esfera de análise: $o$ desenho e as possibilidades que as produtoras e editoras têm a partir da nanotecnologia.

\section{Análise}

Primeiramente procedemos ao reconhecimento dos agentes móveis e dos agentes estáticos, de forma a perceber os agentes intervenientes, as suas proveniências e localização para abordar as formas como o Sul foi retratado. Começámos por identificar a localização geográfica de cada uma das produtoras e editoras para cada um dos videojogos selecionados. Se no jogo de ação/aventura de espionagem Metal Gear Solid IV a produtora Kojima Productions e a editora Konami são ambas japonesas, e se em Metal Gear Rising: Revengeance a produtora Platinum Games e a editora (Konami) também o são, as personagens principais (Snake e Raiden, respetivamente) são ambas norte-americanas. No RPG Deus Ex: Human Revolution, com produtora canadiana e distribuidora japonesa, a personagem central (Adam Jensen) é também norte-americana e a ação principal desenrola-se em Detroit. Em Crysis, título da produtora europeia Crytek e da distribuidora norte-americana Electronic Arts, a personagem principal da primeira entrega (Nomad) e a das duas seguintes (Prophet, Crysis 2 e 3 ) são ambas norte-americanas.

A primeira nota digna de destaque é o facto de todas as personagens principais envolvidas serem norte-americanas, independentemente da localidade da produtora ou da editora do videojogo. Desta forma, Adam Jensen, Snake, Raiden (Jack) e Prophet são todos provenientes dos Estados Unidos da América. Vista a preferência notória por personagens principais de origem norte-americana, procedemos de seguida à análise dos espaços geográficos em que se movem.

O local em que ocorrem os conflitos é digno de relevo dada a pluralidade de lugares. Se em Metal Gear Solid IV os conflitos passam pelo Médio Oriente, América Latina e Europa de Leste, locais tipicamente reconhecidos pelos seus conflitos, em Metal Gear Rising Revengeance os conflitos desencadeiam-se em países africanos (não especificados, desprovendo o continente africano de qualquer sentido identitário no que toca à lógica dos seus Estados e nações), Abcásia, México e Paquistão. De destacar ainda o ataque à sede principal das forças de segurança nos Estados Unidos pelos agentes terroristas, reforçando a ideia que este é o país central a abater.

Em Deus Ex: Human Revolution o local central em que a narrativa se desenrola é Detroit (Estados Unidos), mas com passagens por Hengsha (cidade periférica imaginada nos arredores de Xangai), Montreal (Canadá), Singapura e Panehaea (uma base militar instalada no Oceano Ártico). Tal como na série Metal Gear, o herói desloca-se a estes locais somente para recolher a informação ou os materiais necessários para a resolução dos conflitos na sua cidade. Assim, a deslocação de Adam Jensen para os restantes países nada

Configurações, 12 | 2013 
mais é do que uma mera transição de espaços, pois não é lá que decorre a narrativa principal.

No primeiro Crysis, as forças americanas, equipadas com nanofatos (equipamento militar capaz de melhorar as capacidades de combate), são mobilizadas de forma a eliminar as forças hostis posicionadas na Coreia do Norte, enquanto nas duas restantes entregas o centro dos conflitos situa-se nos Estados Unidos. A invasão alienígena que ocorre em cada um dos títulos da série da Crytek viu o seu fim culminado nos Estados Unidos, numa batalha em que os americanos somente puderam contar consigo mesmos, chegando mesmo à opção de enclausurarem as suas cidades numa redoma, como aconteceu em Crysis 3. Nesta entrega, o fenómeno centra-se na disparidade de poder entre as agências de segurança pertencentes a fundos privados e as agências sob controlo estatal.

Podemos assim observar que os Estados Unidos são vistos como os agentes que se deslocam a países do Sul global para solucionar os problemas que ocorrem nos restantes países do globo. Em suma, os Estados Unidos são vistos como os agentes móveis e o Sul é visto como o agente estático. Mesmo quando os Estados Unidos são vítimas de ataque, como é o caso da cidade de Nova Iorque em Crysis 2 e Crysis 3, cabe aos próprios agentes americanos a resolução desses conflitos, não havendo quaisquer agências ou forças internacionais de segurança que contribuam para a efetiva resolução do conflito. Mais uma vez, o Sul é posto de parte no que diz respeito às suas capacidades de manutenção da paz global.

Num próximo passo visamos perceber que área da nanotecnologia foi retratada em cada um dos videojogos. As três séries, embora diferentes no que respeita ao género, à proveniência das produtoras e das editoras, focaram a nanotecnologia em combinação com as suas aplicações militares. Embora sejam vários os autores que afirmam que o ponto central da nanotecnologia reside na sua combinação com a produção, distribuição e armazenamento energético (Singer, 2005; Smalley, 2005), tem sido através da esfera militar que o investimento tem sido alocado. Na realidade, o sector que nos Estados Unidos mais contribui para a investigação e desenvolvimento em nanotecnologia é o Departamento de Defesa.

41 Mas nem só o aspeto bélico é visível nos videojogos estudados. A questão do melhoramento da saúde física e das condições biológicas do ser humano é tema central em Deus Ex: Human Revolution. Aliás, é notória a dicotomia entre o grupo de indivíduos que apoia a incorporação de inovações assentes na biotecnologia e na nanotecnologia e o daqueles que pretendem manter a condição sapiens e a evolução natural da espécie. Os procedimentos de nanobiotecnologia alastram-se e permitem uma maior esperança de vida, bem como melhorias ao nível da visão e audição. Esta tecnologia permite também gerar uma força sobre-humana.

Mas, se seria de esperar que, com todas estas diferenças na transformação do humano, os sistemas políticos, económicos, sociais, culturais sofressem transformações substanciais, tal não parece acontecer. Assim, um outro aspeto relevante em Deus Ex: Human Revolution diz diretamente respeito à sua linha temporal. A ação tem lugar em 2027, período no qual os Estados Unidos continuam a ser a maior potência económica mundial, assim como a grande força militar. Também o sistema político e económico se mantém inalterável, demonstrando uma lógica de continuidade e não de quebra. Mesmo quando parte da ação se desenrola em território chinês, este país é apresentado de uma forma similar à sua realidade atual. 

aparecimento constante em edifícios, tendas e uniformes, também é evidente a representação do Norte como dominante e a visão do Sul como um espaço de conflito, num meio de caos político e de constante necessidade de apoio militar. $\mathrm{O}$ orgulho nacional ferido, o atraso irremediável dos países, a lentidão dos progressos sociais e económicos são fatores que servem de acendalhas para a revolta do Sul em relação a si e ao Norte e como fonte essencial do conflito. É devido a todas estas formas de instabilidade que surgem grupos de guerrilheiros e terroristas que criam os elementos que perturbam a ordem gerada e trazida pelo Norte global. revolucionária no que toca às suas aplicações, mas também como fonte de manutenção do status quo em termos económicos, políticos e sociais.

\section{Conclusões}

O crescente investimento em nanotecnologia, combinado com o crescimento dos videojogos como forma de entretenimento, tem aberto novas possibilidades de análise. Se os videojogos possuem a capacidade de desafiar as lógicas da física, da química, da economia e da cultura, tal não parece acontecer com os objetos analisados. Se, em termos tecnológicos, as inovações são imensas, especialmente no que toca aos sectores de aplicação, em particular os níveis militar, de saúde e energético, tais inovações não ocorrem no que comporta a mudanças em termos políticos e socioeconómicos. Também a nanotecnologia, aplicada numa vasta amplitude de sectores de aplicação, está confinada às áreas de investimento centrais nos Estados Unidos, dado serem o Departamento de Defesa e o sector de saúde aqueles que consomem os maiores investimentos.

De forma distinta, os videojogos parecem ignorar as lógicas de colonização existentes desde a era moderna, descartando os países do Sul como centros nevrálgicos da ação. A título de exemplo, a dicotomia entre considerar Portugal como ostracizado perante as restantes potências colonizadoras (o que lhe confere uma não pertença como agente da modernidade) e imaginar-se de modo megalómano como o centro irradiador sui generis face a outros colonizadores (Khan, 2011) é claramente refletida no que encontramos nos videojogos analisados: o Sul é ignorado como agente central, e Portugal situa-se nesse conjunto de países ignorados quer como agentes de participação direta, quer como espaço onde decorrem os conflitos. O Sul é destacado somente quando ocorre uma situação em que o agente principal (do Norte) se desloca a um Estado ou nação do Sul de forma a evitar ou a resolver um dado conflito. Assim, países do Sul que não tenham conhecido conflitos militares recentes, tais como Portugal, parecem excluídos do meio dos videojogos.

Outra conclusão que verificamos reside no facto de o próprio Norte criar uma hierarquia rígida. Desta forma, as produtoras europeias e japonesas parecem transferir para os Estados Unidos a capacidade militar, elegendo este país como o garante da paz e o solucionador dos conflitos militares. Quando os conflitos se desenrolam nos Estados Unidos, estes dependem apenas de si mesmos para a sua resolução, não necessitando de apoio internacional; além disso, quando os conflitos passam para territórios quer do Norte, quer do Sul global, também são os elementos daquele país que os resolvem. Assume-se que os Estados Unidos são os mais bem equipados e os mais bem preparados 
para lidar com qualquer problema, desde invasões alienígenas a conflitos militares diretos ou de guerrilha.

Por fim, o género de jogo não apresenta grandes diferenças, uma vez que títulos RPG (Deus Ex: Human Revolution), centrados na narrativa, e storytelling não contribuem com diferentes espaços analíticos em relação a shooters, títulos mais centrados na jogabilidade e não na sua narrativa. Não se revelam, portanto, marcas diferenciais significativas no âmbito do enquadramento das Epistemologias do Sul.

Porém, a discussão em torno da narrativa e da jogabilidade de todos estes títulos não se esgota nesta temática, sendo possível outras formas de análise que diferenciem substancialmente cada um dos títulos, produtoras e editoras.

Outro fenómeno não analisado neste artigo reside nas interações dos MMO e na possibilidade de o jogador escolher as características físicas da sua personagem e o seu sexo, um conjunto de elementos não passíveis de serem controlados no mundo físico. Estas possibilidades, aliadas ao facto de as missões exigirem quase forçosamente a cooperação entre vários jogadores, suscitaria interesse na análise da relação online entre indivíduos do Sul (intra-Sul e Sul-Norte) e do Norte (intra-Norte e Norte-Sul).

\section{BIBLIOGRAFIA}

APPADURAI, Arjun (1988), The Social Life of Things. Commodities in cultural perspective. Cambridge: Cambridge University Press.

BRANDÃO, A. M. \& Araújo, E. R. (2011), Intersecções Identitárias. Famalicão: Edições Húmus.

ARENDT, Hannah (1968), "The crisis in culture", in Between Past and Future: Eight Exercises in Political Thought. London: Penguin Books.

ARRUDA, J. P. (2012), “Das Índias a Pandora. Ensaios sobre a representação do ‘Outro’ na modernidade", Oficina Do CES, (380): 1-24.

BAINBRIDGE, W. S. (2007), "Ethical considerations in the advance of nanotechnology", in Lynn E. Foster (ed.), Nanotechnology - Science, innovation, and opportunity. New Jersey: Prentice Hall, 233-242.

BEN-DAVID (1975), Sociologia da Ciência. Rio de Janeiro: Editora da Fundação Getúlio Vargas.

BENSAUD-VINCENT, Bernardette (2010), “Objects in nanotechnology”, in Ulrich Fiedeler et al. (orgs.), Understanding Nanotechnology. Heidelberg: AKA Verlag, 9-22.

BILLIEUX, J.; VAN DER LINDEN, M.; ACHAB, S.; KHAZAAL, Y.; PARASKEVOPOULOS, L.; ZULLINO, D., \& THORENS, G. (2013), "Why do you play World of Warcraft? An in-depth exploration of selfreported motivations to play online and in-game behaviours in the virtual world of Azeroth", Computers in Human Behavior, 29(1): 103-109.

BORNET, Philippe \& BURGER, Maya (2012), Religions in Play. Games, rituals, and virtual worlds. Zürich: TVZ Theologischer Verlag.

BOURDIEU, Pierre (1999), On Television. New York: New Press. 
BOURDIEU, Pierre (2001), Razões Práticas sobre a Teoria da Acção. Lisboa: Celta Editora.

BOURDIEU, Pierre (2008), Para uma Sociologia da Ciência. Lisboa: Edições 70.

BOWMAN, Diana \& HODGE, Graeme (2007a), “Nanotechnology and public interest dialogue: Some international observations”, Bulletin of Science, Technology \& Society, 27: 118-132.

BOWMAN, Diana M.; HODGE, Graeme A., \& BINKS, Peter (2007b), “Are we really the prey? Nanotechnology as science and science fi ction”, Bulletin of Science, Technology \& Society, 27, 435.

CARBONE, M. B. \& RUFINO, P. (2013), "Introduction: Games and subcultural theory", GAME Games as Media Entertainment, 3: 5-20.

COBB, M. D. \& MACOUBRIE, J. (2004), "Public perceptions about nanotechnology: Risks, benefits and trust", Journal of Nanoparticle Research, 6(4), 395-405.

COBB, M. D. (2005), "Framing effects on public opinion about nanotechnology", Science Communication, 27, 221.

CRICHTON, Michael (2006), Prey. London: HarperCollins Publishers.

DECKER, S. A. \& GAY, J. N. (2011), “Cognitive-bias toward gaming-related words and disinhibition in World of Warcraft gamers", Computers in Human Behavior, 27(2): 798-810.

DEUS, J. D. (1975), A Crítica da Ciência. Rio de Janeiro: Zahar Editores.

DREXLER, E. K. (1990), Engines of Creation. The coming era of nanotechnology. New York: Anchor Books.

DUPUY, J.-P. (2010), “The narrative of lay ethics”, Nanoethics, 4: 153-170.

ELLUL, J. (1967), The Technological Society. New York: Vintage.

ESKELINEN, Markku (2001), "The gaming situation”, Game Studies 1 (1).

FELDMAN, J.; MONTESERIN, A., \& AMANDI, A. (2014), “Detecting students' perception style by using games", Computers \& Education, 71: 14-22.

FERRARI, A. (2010), "Developments in the debate on nanoethics: Traditional approaches and the need for new kinds of analysis", Nanoethics, (4): 27-52.

FIEDELER, Ulrich (2010), "Vision assessment of nanotechnology”, in Ulrich Fiedeler et al. (orgs.), Understanding Nanotechnology. Heidelberg: AKA Verlag, 23-42.

FOLLADORI, Guillermo; INVERNIZZI, Noela, \& ZÁYAGO, Edgar (2009), “Two dimensions of the ethical problems related to nanotechnology", Nanoethics, 3: 121-127.

GERBNER, George (1995), “TV as storyteller”, in GERBNER, Georger, The Electronic Storyteller: Television and the Cultivation of Values. Pennsylvania: University of Pennsylvania.

GIDDENS, A. (2006), O Mundo na Era da Globalização. Lisboa: Editorial Presença.

GOFFMAN, Erving (1979), Gender Advertisements. Cambridge: Macmillan.

GROSFOGUEL, R. (2008), “Para descolonizar os estudos de economia política e os estudos póscoloniais: Transmodernidade, pensamento de fronteira e colonialidade global", Revista Crítica de Ciências Sociais, 80, 115-147.

HABEL, C. \& KOOYMAN, B. (2014), “Agency mechanics: gameplay design in survival horror video games”, Digital Creativity, 25(1): 1-14.

HABERMAS, J. (2006), Técnica e Ciência como "Ideologia". Lisboa: Biblioteca de Filosofia Contemporânea, Edições 70. 
HEIDBRINK, Simone; KNOLL, Tobias, \& WYSOCKI, Jan (2014), “Theorizing religion in digital games - Perspectives and approaches", Heidelberg Journal of religions on the Internet - Religion in Digital Games, 5: 5-50.

HEGEL, Georg (1995), A Razão na História. Lisboa: Edições 70.

HEIDEGGER, Martins (1962), Being and Time. Oxford: Blackwell.

JONES, Richard (2007), Soft Machines. New York: Oxford University Press.

KHAN, Sheila (2011), “O Sul mesmo aqui ao lado: Cartografias identitárias abissais do póscolonialismo português”, in Brandão, A. M. \& Araújo, E. R. (eds.), Intersecções Identitárias. Famalicão: Edições Húmus.

KATZ, Evie; SOLOMON, Fiona; MEE, Wendy, \& LOVEL, Roy (2009), "Evolving scientific research governance in Australia: a case study of engaging interested publics in nanotechnology research", Public Understanding of Science, 18: 531-545.

KULINOWSKI, Kristen (2004), “Nanotechnology: From 'wow' to 'yuck'?”, Bulletin of Science, Technology \& Society, 24: 13-20.

KURZWEIL, Ray (2006), "Nanotech could give global warming a big chill”, Nanotech could give global warming a big chill”, Forbes/Wolfe Nanotech Report, 5, (7).

LEVI-STRAUSS, Claude (1976), O Pensamento Selvagem. São Paulo: Editora Nacional.

LI, D. D.; LIAU, A. K., \& KHOO, A. (2013), "Player-Avatar Identification in video gaming: Concept and measurement", Computers in Human Behavior, 29(1): 257-263.

MACNAGHTEN, P.; KEARNES, M., \& WYNNE, B. (2005), "Nanotechnology, governance, and public deliberation: What role for the social sciences?", Science Communication, 27(2): 268-291.

MACOUBRIE, Jane (2006), "Nanotechnology: Public concerns, reasoning and trust in Government”, Public Understanding of Science, 15: 221.

MARCHAND, A. \& HENNIG-THURAU, T. (2013), "Value creation in the videogame industry: Industry economics, consumer benefi ts, and research opportunities", Journal of Interactive Marketing, 27(3): 141-157.

MARTINS, H. (2006), "Paths to the post human: a very short guide for the perplexed", Configurações, 2: 29-56.

MARTINS, P. R. \& BRAGA, R. (2007), "Nanotecnologia: Promessas e dilemas da Revolução Invisível”, in Sociologia. São Paulo: Editora Scala.

MILBURN, Colin (2005), “Nanowarriors: Military nanotechnology and comic books”, Intertexts 9 (1): 77-103.

MILBURN, C. (2011), "Just for fun: The playful image of nanotechnology", Nano Ethics, 5(2), 223-232.

NERLICH, Brigitte (2005), "From Nautilus to Nanobo(a)ts: The visual construction of nanoscience", AZojono, 1.

NEVES, José Pinheiro (2006), O Apelo do Objecto Técnico. Porto: Campo das Letras.

NORDMANN, Alfred \& MACNAGHTEN, Phil (2010), "Engaging narratives and the limits of lay ethics: Introduction", Nanoethics, 4: 133-140.

NUNES, J. A. (2008), “O resgate da epistemologia”, Revista Crítica de Ciências Sociais, 80: 45-70.

PASSET, Rénée (2002), A Ilusão Neoliberal. Lisboa: Terramar. 
POPPER, Karl R. (1989), Em Busca de um Mundo Melhor. Viseu: Editorial Fragmentos.

PRENSKY, M. (2007), Digital Game-Based Learning. New York: McGraw-Hill.

RIBEIRO, Rita (2011), A Europa na Identidade Nacional. Porto: Edições Afrontamento.

ROCO, M. C. (2007), “Convergence and Integration”, in Lynn E. Foster (ed.), Nanotechnology-

Science, innovation, and opportunity. New Jersey: Prentice Hall, 225-232.

SATWICZ, T. \& STEVENS, R. (2008), "Playing with representations: How do kids make use of quantitative representations in videogames?", International Journal of Computers for Mathematical Learning, 13(3): 179-206.

SANTOS, B. S. (2008), “A filosofia à venda, a douta ignorância e a aposta de Pascal”, Revista Crítica de Ciências Sociais, 80: 11-43.

SHAW, A. (2010), Identity, Identification, and Media Representation in Video Game Play: An audience reception study. PhD Thesis Communication, University of Pennsylvannia.

SENNET, Richard (2007), A Cultura do Novo Capitalismo. Lisboa: Relógio D’Água.

SHELLEY, T. (2009), Nanotechnology: New promises, new dangers. London: Zed Books.

SHELLEY-EGAN, Clare (2010), “The ambivalence of promising technology”, Nanoethics, 4: 183-189.

SHIN, Dong-He (2010), "The dynamic user activities in massive multiplayer online role playing games", International Journal of Human Computer Interaction, 26(4): 317-344.

SIMÕES, Maria João (2005), Política e Tecnologia. Tecnologias da informação e da comunicação e participação política em Portugal. Oeiras: Celta Editora.

SINGER, P.; SALAMANCA-BUENTELLO, F.; PERSAD, D. L.; COURT, E. B.; MARTIN, D. K., \& DAAR, A. S.(2005), "Nanotechnology and the developing world", Nature Nanotechnology, 2(5), 97.

SMALLEY, Richard (2005), "Future global energy prosperity: The Terawatt challenge", Materials Research Society - Symposium X - Frontiers of Materials Research.

SMALLEY, Richard (2007), "Nanotechnology and our energy challenge”, in Lynn E. Foster (org.), Nanotechnology - Science, innovation, and opportunity: New Jersey: Prentice Hall.

TOUMEY, C. (2011), "Seven religious reactions to nanotechnology", Nano Ethics, 5.

WALLERSTEIN, Immanuel (1999), The End of the World as We Know It. Minneapolis: University of Minnesota Press.

WEIBEL, D.; WISSMATH, B.; HABEGGER, S.; STEINER, Y., \& GRONER, R. (2008), "Playing online games against computer vs. human-controlled opponents: Effects on presence, flow, and enjoyment", Computers in Human Behavior, 24(5), 2274-2291.

WOOD, S.; JONES, R., \& GELDART, A. (2007), “The social and economic challenges of nanotechnology", Current, 5.

YOUNG, G. \& WHITTY, M. T. (2010), “Games without frontiers: On the moral and psychological implications of violating taboos within multi-player virtual spaces", Computers in Human Behavior, 26(6): 1228-1236.

ZAGALO, N. (2009), Emoções Interactivas: Do cinema para os videojogos. Coimbra: Grácio Editor.

ZHONG, Z.-J. (2011), “The effects of collective MMORPG (Massively Multiplayer Online RolePlaying Games) play on gamers' online and offl ine social capital", Computers in Human Behavior, 27 (6). 


\section{NOTAS}

1. O investimento realizado no sector transformou-se desde os anos 70, passando de um nicho de mercado a um negócio de blockbusters. A título de exemplo, Call of Duty: Modern Warfare 3 (shooter) obteve quatrocentos milhões de dólares em apenas 24 horas (Marchand \& Hennig-Thurau, 2013), enquanto Gran Theft Auto V (ação/aventura) atingiu a fasquia dos mil milhões de dólares em apenas 48 horas.

2. Referência ao tema da revista GAME dedicada aos estudos dos videojogos intitulada "Video Game Subcultures: Playing at the Periphery of Mainstream Culture".

3. Conceito incorporado na filosofia de Heidegger (1962) que significa "o ser", mas que sobretudo problematiza e compreende o que é "ser".

4. O Presidente Clinton realizou em 1999 um discurso sobre a importância da nanotecnologia enquanto forma de desenvolvimento económico e social.

5. Como no caso dos Massively Multiplayer Online (MMO), em especial nos MMORPG como o World of Warcraft (Zhong, 2011), o Everquest (Weibel et al., 2008), os Final Fantasy XI e XIV, o Age of Conan e o Warhammer (Young \& Whitty, 2010).

6. Como Eskelinen (2001: 7) referiu: "If I throw a ball at you I don't expect you to drop it and wait until it starts telling stories."

7. Se os jogos parecem ter efeitos educacionais em contexto escolar (Rosas et al., 2003), na medida em que encorajam a aplicação de conteúdos escolares ao mundo real (Shin et al., 2010), também revelam o benefício de apresentar conceitos abstratos no contexto de aplicações familiares. Da mesma forma, a motivação dos alunos quando aprendem conteúdos em forma de jogos é maior do que em sistemas educacionais não personalizados (Prensky, 2007). Contudo, o trabalho de Feldman et al. (2014) revela que a aprendizagem e a interiorização de resultados dependem igualmente do estilo e formas de jogabilidade em que elementos, tais como o nível de difi culdade e o género (puzzle, plataforma, RPG, ação) influenciam a imersão e a aprendizagem (Satwicz e Stevens,2008).

8. Sendo o objetivo deste artigo a ligação temática da nanotecnologia com várias aplicações como os videojogos e não o estudo da indústria dos videojogos per si, não se fará a divisão dos videojogos consoante a plataforma.

9. A sucessora da Wii, a Wii U, foi lançada mundialmente ainda durante o final de 2012, enquanto a PlayStation 4 chegou aos consumidores no final de 2013. Não as incluímos na abordagem, de forma a trabalhar unicamente com uma geração de consolas e dado que a nova consola da Microsoft (Xbox One) não teve um lançamento global, mas somente em alguns mercados selecionados, e não tem data de lançamento prevista para os restantes mercados.

10. Um franchise envolve uma linha de produtos que partilham uma série de eventos, narrativas, personagens ou contextos. No universo dos videojogos existe também uma forte presença transmédia, em particular pela criação de novelas gráficas, literatura, filmes (animados) ou séries televisivas, para além da comercialização de merchandising (Habel \& Kooyman, 2014). 


\section{RESUMOS}

A nanotecnologia é apresentada como uma tecnologia emergente capaz de revolucionar o mundo em que vivemos. A sua convergência com outros sectores tecnológicos como a biotecnologia e as tecnologias de informação e com sectores de aplicação, como a área da energia, produção alimentar, saúde, esfera militar, constitui-a como um campo do conhecimento multidisciplinar e de aplicação interindustrial. Questionamos assim qual o contributo dos videojogos e a forma como esta indústria retrata a nanotecnologia de forma a perceber a criação dos atuais paradigmas tecnológicos, bem como se servem os videojogos analisados para contestar os paradigmas tecnológicos e culturais vigentes. Este artigo visa identificar a forma como a nanotecnologia é retratada em alguns videojogos da presente geração de consolas (2005-2013) nos principais géneros - Ação/Aventura, First Person Shooter (FPS) e Role Playing Games (RPG) -, estimando também quais os papéis que são atribuídos pelas diferentes produtoras e editoras ao conjunto das suas personagens. Assim, a partir das Epistemologias do Sul, pretendemos demonstrar de que forma a nanotecnologia se associa aos sectores de aplicação, mas também que configurações geopolíticas são representadas em cada um dos videojogos analisados. O objetivo deste texto assenta na criação de um modelo de análise baseado nas Epistemologias do Sul que pudesse compreender a forma como uma tecnologia é retratada num meio marcadamente produzido nas indústrias do Norte e que não permite as análises de conteúdo ou de discurso mais tradicionais. A partir da narrativa de cada um dos videojogos estudados, criámos um modelo que integrou as conclusões nas perspetivas teóricas abordadas. A incorporação da nanotecnologia nos videojogos analisados revela a relação a que o Sul está sujeito: ignorado, nunca visto como o herói, mas sempre como um espaço envolto em conflitualidade a que ele próprio parece estar inerente.

Nanotechnology is presented as an emerging technology capable of revolutionizing the world in which we live. Its convergence with other technology sectors such as biotechnology and information technologies and with sectors of application such as energy, food production, health and the military, constitute it as a field of multidisciplinary knowledge with inter-industrial applications. We set out to identify the contribution of video games and the way the industry portrays nanotechnology in order to understand the creation of the current technological paradigms and to see if the video games studied challenge the prevailing technological and cultural paradigms. We describe how nanotechnology is portrayed in some video games created in the present generation of consoles (2005-2013) in the major genres - Action / Adventure, First Person Shooter (FPS) and Role Playing Games(RPG). We also estimate what roles are assigned by different developers and publishers to their characters. Thus, guided by the Epistemologies of the South framework we try to show how nanotechnology is associated with the sectors of application, and which geopolitical configurations are represented in the video games analyzed. The objective of this text rests on the creation of an analysis model based on the Epistemologies of the South that can understand how a given technology is portrayed in a medium largely produced in industries in the North and that rejects the traditional content and discourse analysis. We used the narrative of each video game to establish a model that incorporated the conclusions in the theoretical perspectives. The incorporation of nanotechnology in the video 
games reveals how the South is seen, i.e. sometimes ignored, never as the hero, always as a place engulfed in a conflict that seems inherent to it.

La nanotechnologie est présentée comme une technologie émergente capable de révolutionner le monde dans lequel nous vivons. Sa convergence avec d'autres secteurs technologiques comme les technologies de l'information et la biotechnologie et avec des secteurs d'application tels que le domaine de l'énergie, la production alimentaire, la santé et le secteur militaire en font un domaine de connaissances pluridisciplinaires et d'inter-applications industrielles. Nous questionnons la contribution des jeux vidéo et la façon dont cette industrie imagine la nanotechnologie afin de comprendre la création des paradigmes technologiques actuels et comment elle se sert des jeux vidéo analysés pour défier les paradigmes technologiques et culturels en vigueur. Cet article vise à déterminer comment la nanotechnologie est représentée dans les jeux vidéo de cette génération de consoles (2005-2013) dans les principaux genres action / aventure, first person shooter (FPS) et jeux de rôle (RPG) - mais également estimer les rôles qui sont attribués par les différents développeurs et éditeurs à leurs personnages. Ainsi, à partir des épistémologies du Sud, nous visons à démontrer comment la nanotechnologie est associée aux secteurs d'application, mais également que les configurations géopolitiques sont représentées dans chacun des jeux vidéo analysés. L'objectif de ce texte est la création d'un modèle d'analyse basé sur les épistémologies du Sud, qui pourrait comprendre comment la technologie est dépeinte dans un moyen de communication fortement produit par las industries du Nord, qui interdit les analyses de contenu et de discours plus traditionnel. À partir de la narration de chaque jeu vidéo étudié, nous avons créé un modèle qui intègre les résultats abordés dans les perspectives théoriques. L'intégration de la nanotechnologie dans les jeux vidéo révèle la relation à laquelle le Sud est soumise : parfois ignoré, jamais le héros mais plutôt comme des espaces impliqués dans des conflits dont il semble lui-même inhérent.

\section{ÍNDICE}

Palavras-chave: Nanotecnologia, Epistemologias do Sul, videojogos, jogabilidade, storytelling

Keywords: Nanotechnology, Epistemologies of the South, video games, gameplay, s torytelling

Mots-clés: nanotechnologie, épistémologies du Sud, jeux vidéo, jouabilité, narration

\section{AUTOR}

\section{RUI VIEIRA CRUZ}

Centro de Investigação em Ciências Sociais, Universidade do Minho

rmvcruz@gmail.com 